\title{
A let-7b binding site SNP in the 3'-UTR of the Bcl-xL gene enhances resistance to 5-fluorouracil and doxorubicin in breast cancer cells
}

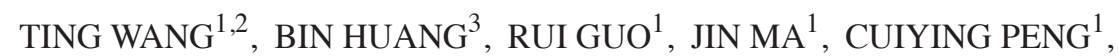 \\ XUYU ZU ${ }^{3}$, HUIFANG TANG ${ }^{3}$ and XIAOYONG LEI ${ }^{1}$ \\ ${ }^{1}$ Institute of Pharmacy and Pharmacology; ${ }^{2}$ Department of Pharmacy, The Second Affiliated Hospital; \\ ${ }^{3}$ Department of Pharmacy, The First Affiliated Hospital, University of South China, Hengyang, Hunan 421001, P.R. China
}

Received February 10, 2014; Accepted December 8, 2014

DOI: $10.3892 / \mathrm{ol} .2015 .2938$

\begin{abstract}
The development of acquired resistance to chemotherapy is a major obstacle in the successful treatment of cancer. In breast cancer cells, B-cell lymphoma-extra large (Bcl-xL) is involved in the development of resistance to various chemotherapeutic agents; therefore, preliminary biological prediction was performed to identify a putative binding site for let-7b in the 3'-untranslated region (UTR) of the Bcl-xL gene and a single nucleotide polymorphism (SNP) within this binding region. The present study investigated the association between the SNP rs3208684 A>C and chemotherapeutic agent resistance in breast cancer cells. The data indicated that let-7b negatively regulates the expression of $\mathrm{Bcl}-\mathrm{xL}$ and appears to sensitize MCF-7 cells to the chemotherapeutic agents 5-fluorouracil (5-FU) and doxorubicin. Furthermore, the SNP rs3208684 $\mathrm{A}>\mathrm{C}$ was demonstrated to enhance $\mathrm{Bcl}-\mathrm{xL}$ protein expression by disrupting the binding of let-7b to the 3 '-UTR of Bcl-xL and, in MCF-7 cells, overexpression of let-7b in the presence of a mutant Bcl-xL 3'-UTR (C allele) significantly increased 5-FU and doxorubicin resistance. Thus, the results of the present study demonstrate that the SNP rs3208684 A>C may upregulate $\mathrm{Bcl}-\mathrm{xL}$ protein expression and enhance the resistance of the MCF-7 cells to 5-FU and doxorubicin by decreasing the binding of let-7b to the 3'-UTR of Bcl-xL.
\end{abstract}

\section{Introduction}

Breast cancer is the leading cause of cancer-related mortality in females, worldwide (1). In the previous two decades, the incidence rate of breast cancer has increased at an average rate

Correspondence to: Professor Xiaoyong Lei, Institute of Pharmacy and Pharmacology, University of South China, 28 Changsheng Road, Hengyang, Hunan 421001, P.R. China

E-mail: leixiaoyong@aliyun.com

Key words: let-7b, B-cell lymphoma-extra large, single nucleotide polymorphism, resistance, breast cancer of $3.1 \%$ per year and the mortality rate has increased at an average rate of $1.8 \%$ per year (2). Chemotherapy is an important adjuvant systemic therapeutic approach for the successful treatment of breast cancer (3) and, during early-stage breast cancer, has been demonstrated to improve survival rate (4).

MicroRNAs (miRNAs) have previously been identified as important regulators of a number of key genes associated with chemoresistance $(5,6)$. miRNAs are a class of endogenous, 18 to 25-nucleotide long, non-coding RNAs, which regulate gene expression at the post-transcription level $(7,8)$. As a recognition mechanism, miRNAs complementarily pair to the 3'-untranslated region (UTR) of their target mRNAs, resulting in decreased translational efficiency and/or decreased mRNA expression levels (9-11). It has previously been reported that miRNAs commonly deregulate gene expression levels in specific types of human cancer, and may serve as oncogenes or tumor suppressors $(12,13)$. However, dysregulated miRNAs appear to be associated with every aspect of the cancer-related biological process, including tumor progression, invasion and metastasis, as well as the acquisition of resistance to various chemotherapeutic agents $(14,15)$. Previous studies have indicated that single nucleotide polymorphisms (SNPs) occurring in or near miRNA binding sites may be associated with tumor susceptibility and chemotherapeutic response in humans (6,17-19).

B-cell lymphoma-extra large (Bcl-xL) belongs to the $\mathrm{Bcl}-2$ protein family and appears to confer resistance to apoptosis, thereby reducing the effectiveness of chemotherapy (20). It has previously been reported that overexpression of Bcl-2 and Bcl-xL increases resistance to totaxol and etoposide administration in MCF-7 cells (21), whereas their downregulation sensitizes MCF-7 and MDA-MB-231 cells to doxorubicin, paclitaxel and cyclophosphamide administration (22). In the present study, we hypothesized that SNPs located in let-7b binding sites of the Bcl-xL gene 3'-UTR may regulate Bcl-xL expression, thus, increasing cellular resistance to chemotherapeutic agents in breast cancer cells. To investigate this hypothesis, bioinformatic analyses were performed to identify SNPs in the 3'-UTR of the Bcl-xL gene. We then functionally validated SNP rs3208684 A>C, which is located in the let-7b binding site in the 3'UTR of the Bcl-xL gene. 


\section{Materials and methods}

SNP selection. To predict putative miRNA binding sites in the Bcl-xL 3'-UTR, microrna.org (http://www.microrna.org/microrna/home.do), PicTar (http://pictar.mdc-berlin.de/cgi-bin/PicTar_vertebrate.cgi) and TargetScan version 6.2 (http://www.targetscan.org/) were used. Furthermore, the National Center for Biotechnology Information SNP database (dbSNP; http://www.ncbi.nlm.nih.gov/SNP) was used to identify SNPs within putative miRNA target sites in the 3'-UTR of Bcl-xL. The search was focused on the miRNA seed region, as the seed sequence nucleates interaction between the miRNA and the complementary Bcl-xL mRNA target region, and is the predominant determinant for successful miRNA targeting.

Cell culture and transfections. The human breast cancer cell line, MCF-7 (Cell Bank of the Chinese Academy of Sciences, Shanghai, China) was cultured in Dulbecco's modified Eagle medium (HyClone Laboratories, Inc., Logan, UT, USA) with $10 \%$ fetal bovine serum (HyClone Laboratories, Inc.) at $37^{\circ} \mathrm{C}$ and $5 \% \mathrm{CO}_{2}$. Upon reaching $70 \%$ confluence, the MCF-7 cells $\left(5 \times 10^{3}\right.$ cells per well) were transfected with let-7b mimics or control miRNA mimics [normal control (NC)] (GenePharma Co., Ltd, Shanghai, China), or wild-type Bcl-xL (WT-Bcl-xL), mutant Bcl-xL (Mut-Bcl-xL) or vector alone (pcDNA3.1) (Invitrogen Life Technologies, Shanghai, China) using Lipofectamine ${ }^{\circledR} 2000$ transfection reagent (Invitrogen Life Technologies, Carlsbad, CA, USA), according to the manufacturer's instructions.

Luciferase reporter assay. To conduct the luciferase reporter assay, HEK293T cells (Land Co., Ltd, Guangzhou, China) were seeded onto 24 -well plates $\left(2 \times 10^{4}\right.$ cells per well). In each well, HEK293T cells were transfected with $0.5 \mu \mathrm{g} \mathrm{Bcl-xL}$ 3'-UTR luciferase reporter plasmids containing A or C alleles (Land Co., Ltd) and $100 \mathrm{nM}$ let-7b mimics, let-7b inhibitor or NC inhibitor using Lipofectamine 2000 (Invitrogen Life Technologies), according to the manufacturer's instructions. At $48 \mathrm{~h}$ post-transfection, the cell lysates were collected and a dual luciferase reporter assay system was used to measure firefly and Renilla luciferase activity (Promega Corporation, Madison, WI, USA); relative luciferase activity was calculated by normalizing the Renilla luciferase activity to the firefly luciferase activity.

Western blot analysis. The cell lysates were separated on $10 \%$ SDS-PAGE and transferred to polyvinylidene fluoride membranes (EMD Millipore Co., Hayward, CA, USA). The membranes were blocked with $5 \%$ skimmed milk for $1 \mathrm{~h}$ and incubated with rabbit monoclonal anti-Bcl-xL (cat. no. 2764), anti-Bcl-2-associated X protein (Bax) (cat. no. 5023) and anti- $\beta$-actin (cat. no. 4970) (Cell Signaling Technology, Inc., Danvers, MA, USA) antibodies at dilutions of 1:1,000 overnight at $4^{\circ} \mathrm{C}$, respectively. Subsequently, the membranes were incubated with a polyclonal goat anti-rabbit horseradish peroxidase-conjugated secondary antibody (dilution, 1:4,000; cat. no. 7074; Cell Signaling Technology, Inc.) for $1 \mathrm{~h}$. Finally, enhanced chemiluminescence (Enhanced Chemiluminescence Western Blotting kit; Amersham Biosciences, Piscataway, NJ, USA) was used to visualize the results and $\beta$-actin was used as internal control.

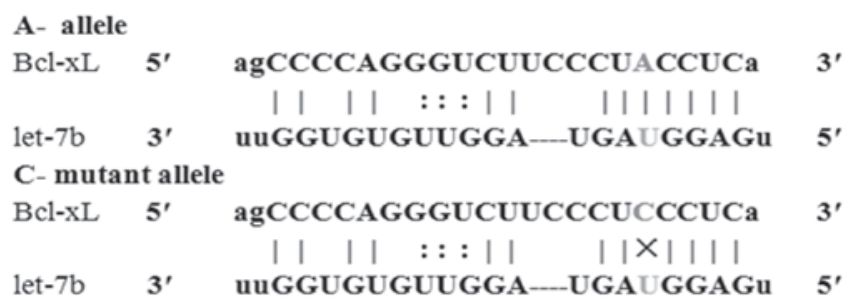

Figure 1. A let-7b SNP binding site in the Bcl-xL 3'-untranslated region. The SNP (rs3208684 A>C) occurs in the 6-bp seed sequence of complementarity at the 5' end of let-7b. SNP, single nucleotide polymorphism; Bcl-xL, B-cell lymphoma-extra large.

3-(4, 5-dimethylthiazol-2-yl)-2,5-diphenyltetrazolium bromide(MTT) assay. Cell viability was detected by performing an MTT (Sigma Aldrich, St. Louis, MO, USA) assay. MCF-7 cells were transfected prior to treatment with $0,6,60,600$, 6,000 and 60,000 $\mu \mathrm{M} 5$-fluorouracil (5-FU) (Sigma-Aldrich) or $0.0,0.25,0.5,1.0,2.0$ and $4.0 \mu \mathrm{M}$ doxorubicin (ADM) (Sigma-Aldrich) for $48 \mathrm{~h}$. Following a 4-h re-incubation with 10\% MTT solution, $150 \mu \mathrm{l}$ DMSO (Sigma-Aldrich) was added to solubilize the resultant formazan crystals. The absorbance of the plate was measured in a microplate reader reader (ELX-800, Bio-Tek Instruments, Inc., Winooski, VT, USA) at a wavelength of $570 \mathrm{~nm}$, with a reference wavelength of $650 \mathrm{~nm}$, and the results were expressed as the percentage of absorbance relative to the untreated controls.

Statistical analysis. Statistical analyses were performed using GraphPad Prism software (version 5.0; GraphPad Software Inc., La Jolla, CA, USA) and Student's t-test. Data are expressed as the mean \pm standard deviation and $\mathrm{P}<0.05$ was considered to indicate a statistically significant difference.

\section{Results}

Identification of let-7b SNP binding sites in the Bcl-xL 3'-UTR. To identify possible miRNA binding sites in the 3 '-UTR of the Bcl-xL gene, bioinformatic analysis was performed using three online prediction programs (PicTar, TargetScan and microrna.org). According to the putatively identified miRNA binding sites combined with information from the dbSNP database, it was identified that the Bcl-xL 3'-UTR SNP rs3208684 $\mathrm{A}>\mathrm{C}$ is located within a predicted miRNA binding site for let-7b (Fig. 1). These results indicate that the $\mathrm{C}$ allele forms a non-perfect pairing with the let-7b miRNA seed and, thus, may escape let-7b-mediated regulation.

Let-7b negatively regulates the protein expression levels of Bcl-xL and sensitizes MCF-7 cells to 5-FU and ADM. Bioinformatic analysis identified a potential binding site of let-7b in the 3'-UTR of the Bcl-xL gene and a dual-luciferase reporter was performed to determine whether let-7b binds at this putative binding site. The full length Bcl-xL 3'-UTR was cloned into the psiCHECK-2 vector (Fig. 2A) and this psiCHECK2-WT-Bcl-xL 3'-UTR vector was subsequently cotransfected into HEK293T cells with let-7b mimics, NC, let-7b inhibitor or inhibitor NC. As indicated in Fig. 2B, luciferase activity was significantly suppressed in the presence 
A

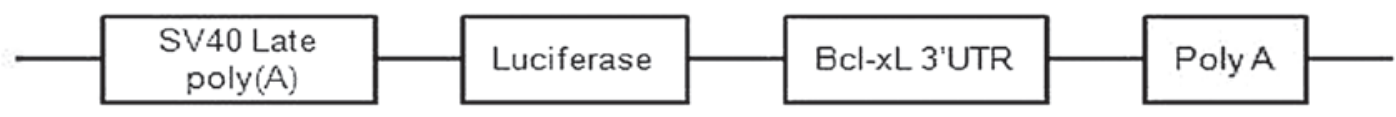

B

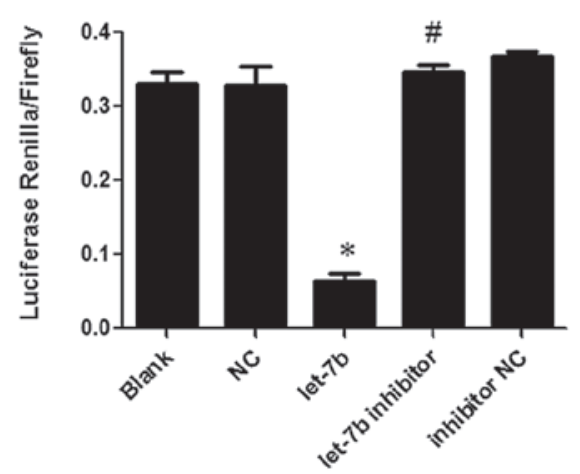

D

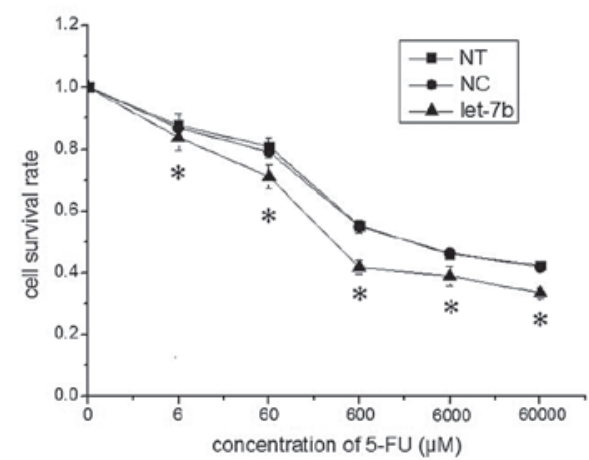

C
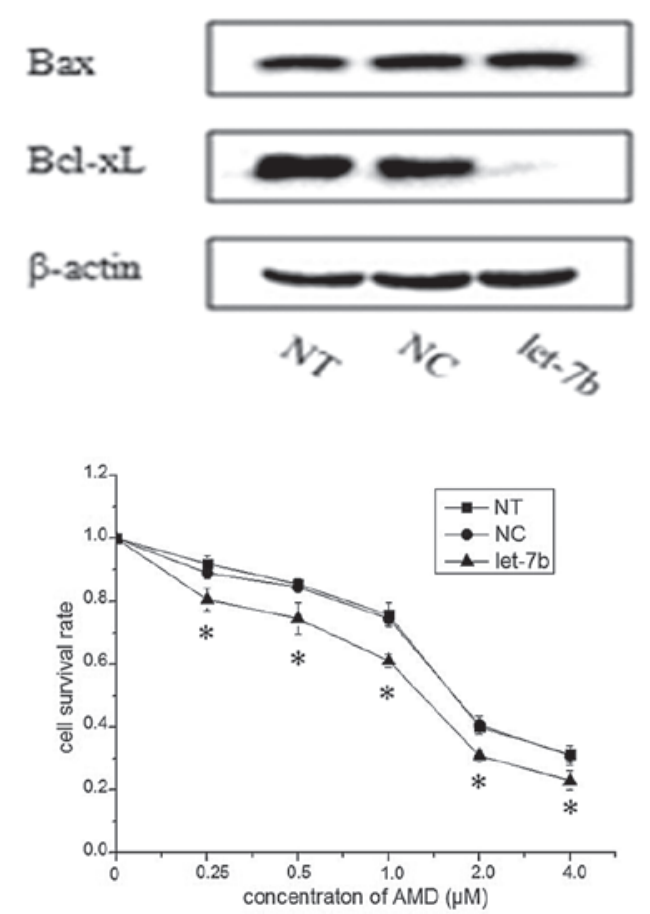

Figure 2. Let-7b negatively regulates the expression of Bcl-xL and sensitizes MCF-7 cells to 5-FU and ADM. (A) Bcl-xL 3'-UTR was cloned into the psiCHECK-2 vector. (B) Luciferase reporter plasmids containing the wild-type Bcl-xL 3'-UTR were transfected in HEK293T cells with $100 \mathrm{nM}$ let-7b, NC, let-7b inhibitor or inhibitor $\mathrm{NC}$ and luciferase expression was measured $48 \mathrm{~h}$ after transfection. The data are represented as the mean \pm standard deviation (SD) of a minimum of three independent transfections analyses. ${ }^{*} \mathrm{P}<0.01$ vs. NC; ${ }^{~} \mathrm{P}>0.05$ vs. inhibitor NC. (C) Let-7b mimics were transfected into MCF-7 cells, and, $48 \mathrm{~h}$ after transfection, cells lysates were prepared and subjected to western blot analysis. NT and NC were used as negative controls, and the data are represented as the mean \pm SD of three independent analyses. (D) MCF-7 cells were transfected with let-7b mimics or NC and treated with different concentrations of 5-FU or ADM. "P<0.05 vs. NC. The cell survival rate was determined by performing an MTT assay and the data are represented as the mean \pm SD of a minimum of three independent transfections analyses. ADM, doxorubicin; Bcl, B-cell lymphoma-extra large; FU, fluorouracil; let-7b, let-7b mimic; MTT, 3-(4, 5-dimethylthiazol-2-yl)-2,5-diphenyltetrazolium bromide; NC, normal control (microRNA mimic); NT, non-transfected cells; SV40 late poly(A), Simian virus 40 late polyadenylation (A); UTR, untranslated region.

of let-7b mimics compared with the $\mathrm{NC}(\mathrm{P}<0.01)$, whereas the luciferase activity displayed no significant difference in cotransfection rate between the let-7b inhibitor and $\mathrm{NC}$ inhibitor groups $(\mathrm{P}>0.05)$.

To exert their function, miRNAs suppress the expression of their target genes (23). To verify whether Bcl-xL is a target of the miRNA let-7b, Bcl-xL protein expression levels were analyzed in response to enforced expression of let-7b in MCF-7 cells. Following transfection with let-7b mimics or NC, MCF-7 cells were analyzed by performing western blot analysis and it was determined that overexpression of let- $7 \mathrm{~b}$ significantly inhibited endogenous Bcl-xL protein expression levels. However, overexpression of let-7b had no effect on Bax protein expression levels (Fig. 2C). These biochemical findings indicate that let-7b is a posttranscriptional regulator of Bcl-xL expression in breast cancer cells.

To evaluate the effect of let-7b on the response of MCF-7 cells to 5-FU and ADM treatment, let-7b mimics or NC were transfected into MCF-7 cells and the sensitivity of these mimic-transfected cells to different concentrations of 5-FU or ADM was determined. The MTT assay indicated that MCF-7 cells overexpressing let-7b were significantly more sensitive to 5-FU and ADM, compared with the control cells ( $\mathrm{P}<0.05$; Fig. 2D).

SNP rs3208684 A>C increases Bcl-xL expression levels by interfering with let-7b function. As SNP rs3208684 A $>C$ is located within the let-7b seed binding site, we hypothesized that this SNP may result in differential regulation of Bcl-xL induced by let-7b due to the differential binding affinity of let-7b for the two Bcl-xL 3'-UTR genotypes. To investigate this hypothesis, the wild-type (containing the A allele) and mutant (containing the C allele) Bcl-xL 3'-UTRs were cloned into the dual-luciferase psiCHECK-2 reporter vector, and HEK293T cells were co-transfected with let-7b mimics or NC. It was identified that luciferase activity significantly decreased in the presence of psiCHECK2-WT-Bcl-xL 3'-UTR plasmids $(\mathrm{P}<0.01)$ but did not change in the presence of psiCHECK2-Mut-Bcl-xL 3'-UTR plasmids $(\mathrm{P}>0.05)$. These data indicate that the SNP rs3208684 A $>$ C may affect let-7b binding to the Bcl-xL 3'-UTR (Fig. 3A).

To determine the effect of the SNP rs3208684 $\mathrm{A}>\mathrm{C}$ on let-7b-mediated regulation of $\mathrm{Bcl}-\mathrm{xL}$ expression, $\mathrm{Bcl}-\mathrm{xL}$ gene expression constructs containing WT-Bcl-xL and Mut-Bcl-xL 
A

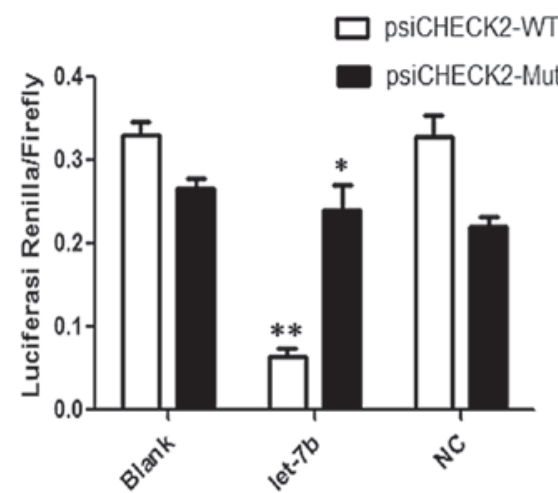

B

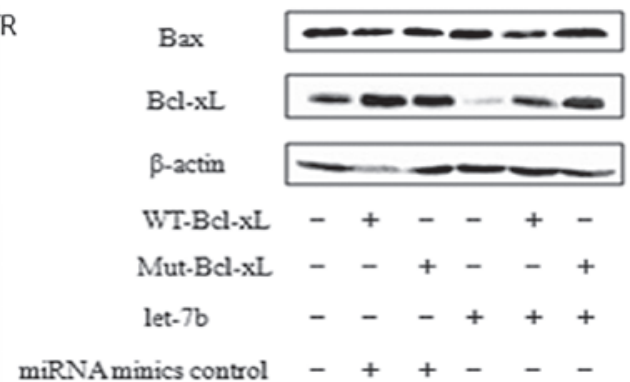

Figure 3. Single nucleotide polymorphism rs3208684 increases Bcl-xL expression by interfering with let-7b function. (A) Luciferase reporter plasmids containing wild-type (psiCHECK2-WT-Bcl-xL-3'-UTR) and mutant (psiCHECK2-Mut-Bcl-xL-3'-UTR) Bcl-xL 3'-UTRs were co-transfected with let-7b mimics or NC into HEK293T cells and luciferase expression was measured $48 \mathrm{~h}$ after transfection. The data are represented as the mean \pm standard deviation of a minimum of three independent transfections analyses. (B) Bcl-xL gene containing a WT-Bcl-xL or Mut-Bcl-xL 3'-UTR were co-transfected into MCF-7 cells with let-7b. Cell lysates were prepared and subjected to western blot analysis $48 \mathrm{~h}$ after transfection. ${ }^{*} \mathrm{P}>0.05 \mathrm{vs}$. $\mathrm{NC}$; ${ }^{* *} \mathrm{P}<0.001 \mathrm{vs}$. $\mathrm{NC}$. Bax, Bcl-2-associated protein X; Bcl, B-cell lymphoma-extra large; miRNA, micro RNA; mut, mutant; NC, normal control (miRNA mimic); UTR, untranslated region; WT wild-type.
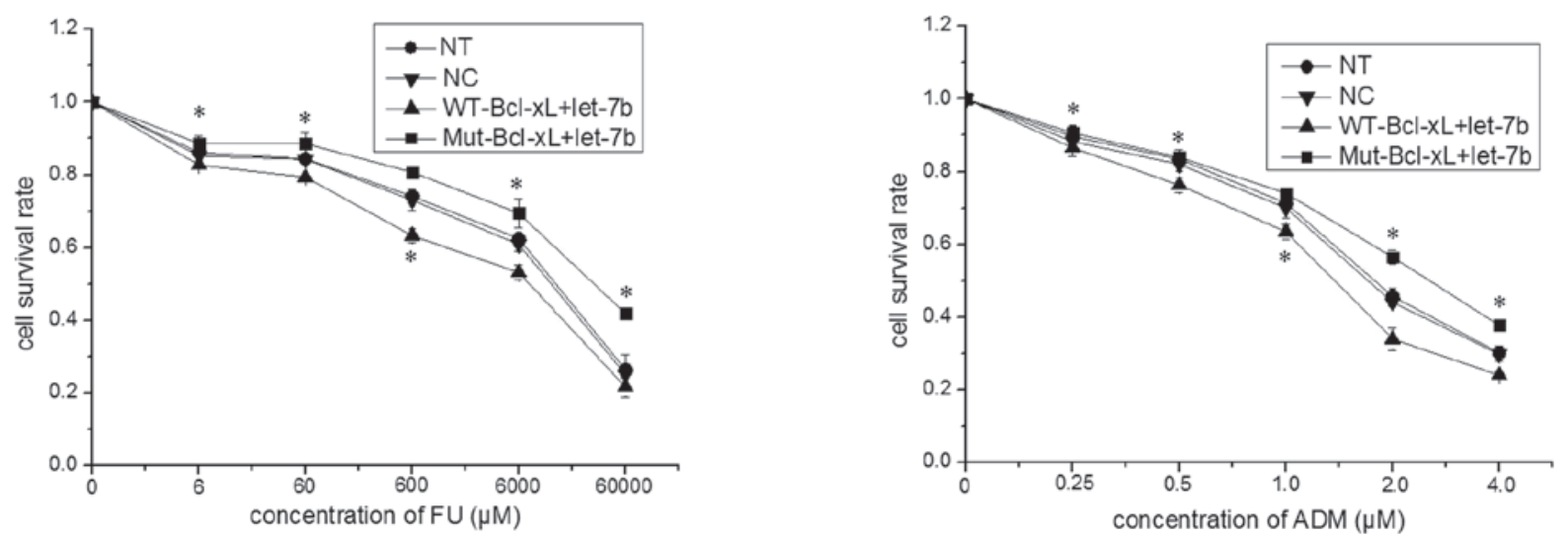

Figure 4. Single nucleotide polymorphism rs3208684 A>C enhances the resistance of MCF-7 cells to 5-FU and ADM. The expression constructs of the full-length Bcl-xL gene containing A (WT-Bcl-xL) or C (Mut-Bcl-xL) were co-transfected with let-7b into MCF-7 cells and, $24 \mathrm{~h}$ after transfection, the MCF-7 cells were treated with various concentrations of 5-FU or ADM for $48 \mathrm{~h}$. The cell survival rate was determined by performing an MTT assay and the data are expressed as the mean \pm standard deviation of a minimum of three independent transfection analyses. ${ }^{*} \mathrm{P}<0.05 \mathrm{vs}$. WT-Bcl-xL+let-7b. ADM, doxorubicin; Bcl, B-cell lymphoma-extra large; FU, fluorouracil; MTT, 3-(4, 5-dimethylthiazol-2-yl)-2,5-diphenyltetrazolium bromide; mut, mutant; NT, non-transfected cells; WT wild-type; NC, normal control (miRNA mimic).

were generated and co-transfected into MCF-7 cells with let-7b mimics. As indicated in Fig. 3B, overexpression of let-7b caused a decrease in $\mathrm{Bcl}-\mathrm{xL}$ gene expression in the presence of WT-Bcl-xL compared with Mut-Bcl-xL; however, the SNP rs3208684 A $>C$ did not change Bax protein expression levels. Thus, the present study proposes that variation in the SNP rs3208684 A>C may mediate the upregulation of Bcl-xL protein expression by interfering with the binding of let- $7 \mathrm{~b}$ to the 3'-UTR of Bcl-xL in breast cancer cells.

SNP rs3208684 A>C enhances the resistance of MCF-7 cells to 5-FU and $A D M$. The current study investigated whether the presence of an SNP in the let-7b binding site of the Bcl-xL 3'-UTR results in resistance to chemotherapeutic agents. WT-Bcl-xL or Mut-Bcl-xL were co-transfected into MCF-7 cells with let-7b mimic and, after $24 \mathrm{~h}$, these transfected MCF-7 cells were treated with various concentrations of 5-FU or ADM for an additional $48 \mathrm{~h}$. As indicated in Fig. 4, the survival rate of MCF-7 cells transfected with Mut-Bcl-xL and let-7b was significantly higher than that in MCF-7 cells transfected with WT-Bcl-xL and let-7b $(\mathrm{P}<0.05)$. Thus, the SNP rs3208684 A $>C$ in the let-7b binding site of Bcl-xL 3'-UTR appears to result in let-7b-associated 5-FU and ADM resistance in MCF-7 cells.

\section{Discussion}

$\mathrm{Bcl}-\mathrm{xL}$ is one of two protein products of the Bcl211 gene (24) and is a primary antiapoptotic factor, which has been recognized to mediate chemotherapeutic agent resistance. Previously, Bcl-xL and Bax were identified as key factors in the regulation of apoptosis by homodimerization and heterodimerization (25). The human let-7 family is classified as a tumor suppressor family in human cancer (26) and a number of previous studies have indicated that the expression of members of the let-7 family are significantly downregulated in various types of cancer (27), including breast cancer; furthermore, this downregulation is associated with a poorer clinical outcome (28). In the present study, let-7b was demonstrated to target $\mathrm{Bcl}-\mathrm{xL}$, resulting in its 
downregulation in MCF-7 cells. In addition, the present study indicated that let-7b enhances the sensitivity of MCF-7 cells to ADM and 5-FU. These results indicate that let-7b overexpression may enhance cellular sensitivity to 5-FU and ADM via the repression Bcl-xL expression in MCF-7 cells.

In a number of critical genes, SNPs at or adjacent to miRNA binding sites are associated with the chemotherapeutic response of a tumor via the disturbance or obstruction of miRNA binding (29-32). The possible causes of this are the SNPs located in the 'seed' regions at the 3'-UTRs of human genes involved in multiple pathways such as cell proliferation, cell death, stress resistance which are likely to affect miRNA-target interaction and target expression accordingly (31). The dual-luciferase reporter assay conducted in the current study revealed that the presence of rs3208684 A-3'-UTR in the let-7b binding site of the Bcl-xL 3'-UTR significantly reduces the expression of luciferase compared with the presence of rs3208684 C-3'-UTR. This is consistent with the initial bioinformatic analysis, which indicated a functional interaction between let-7b and $\mathrm{Bcl}-\mathrm{xL}$ mRNA. Additionally, the transfection of MCF-7 cells with rs3208684 A-3'-UTR and let-7b mimic demonstrated significant inhibition of Bcl-xL expression, and the SNP rs3208684 A>C was identified to cause 5-FU and ADM resistance in MCF-7 cells. Thus, these results indicate that the occurrence of an SNP in rs3208684 A-3'-UTR of Bcl-xL may contribute to the alteration of cellular resistance to 5-FU and ADM.

In conclusion, the present study, demonstrated that let-7b may enhance the sensitivity of MCF-7 cells to 5-FU and ADM by regulating Bcl-xL expression. The SNP rs3208684 A to C may inhibit the interaction between let-7b and Bcl-xL 3'-UTR, resulting in higher Bcl-xL expression, as well as cellular resistance to 5-FU and ADM. Thus, we propose that the SNP rs3208684 A>C may be a potential marker for personalized therapeutic approaches. Furthermore, these results provide insight into a potential novel chemotherapeutic strategy for breast cancer by combining let-7b with currently used chemotherapeutic agents.

\section{Acknowledgements}

This present study was supported by the National Natural Science Foundation of China (grant nos. 81372579, 31371277 and 30900625), the Colleges and Universities Innovation Platform Open Fund Project of Hunan Province of China (grant no. 13K084), the Science and Technology Program fund of Hunan Province of China (grant no. 212FJ2016) and Provincial Key Discipline for Pharmacy of Hunan Province of China during the Twelfth Five-year Plan Period.

\section{References}

1. Jemal A, Bray F, Center M M, Ferlay J, Ward E and Forman D: Global cancer statistics. CA Cancer J Clin 61: 69-90, 2011.

2. Forouzanfar MH, Foreman KJ, Delossantos AM, et al: Breast and cervical cancer in 187 countries between 1980 and 2010: a systematic analysis. Lancet 378: 1461-1484, 2011.

3. Oostendorp LJ, Stalmeier PF, Donders AR, et al: Efficacy and safety of palliative chemotherapy for patients with advanced breast cancer pretreated with anthracyclines and taxanes: a systematic review. Lancet Oncol 12: 1053-1061, 2011.

4. Perez E and Muss HB: Optimizing adjuvant chemotherapy in early-stage breast cancer. Oncology (Williston Park) 19: 1759-1767; discussion 1768, 1772-1774, 1777-1778, 2005.
5. Sarkar FH, Li Y, Wang Z, Kong D and Ali S: Implication of microRNAs in drug resistance for designing novel cancer therapy. Drug Resist Updat 13: 57-66, 2010.

6. Majumder S and Jacob ST: Emerging role of microRNAs in drug-resistant breast cancer. Gene Expr 15: 141-151, 2011.

7. Garzon R, Marcucci G and Croce CM: Targeting microRNAs in cancer: rationale, strategies and challenges. Nat Rev Drug Discov 9: 775-789, 2010.

8. Lee S and Vasudevan S: Post-transcriptional stimulation of gene expression by microRNAs. Adv Exp Med Biol 768: 97-126, 2013.

9. Bartel DP: MicroRNAs: target recognition and regulatory functions. Cell 136: 215-233, 2009.

10. Guo H, Ingolia NT, Weissman JS and Bartel DP: Mammalian microRNAs predominantly act to decrease target mRNA levels. Nature 466: 835-840, 2010.

11. Djuranovic S, Nahvi A and Green R: miRNA-mediated gene silencing by translational repression followed by mRNA deadenylation and decay. Science 336: 237-240, 2012.

12. Lujambio A and Lowe SW: The microcosmos of cancer. Nature 482: 347-355, 2012

13. Singh R and Mo YY: Role of microRNAs in breast cancer. Cancer Biol Ther 14: 201-212, 2013.

14. Sandhu S and Garzon R: Potential applications of microRNAs in cancer diagnosis, prognosis, and treatment. Semin Oncol 38: 781-787, 2011.

15. Cho WC: MicroRNAs as therapeutic targets and their potential applications in cancer therapy. Expert Opin Ther Targets 16: 747-759, 2012.

16. Mishra PJ, Humeniuk R, Mishra PJ, et al: A miR-24 microRNA binding-site polymorphism in dihydrofolate reductase gene leads to methotrexate resistance. Proc Natl Acad Sci USA 104: 13513-13518, 2007.

17. Saetrom P, Biesinger J, Li SM, et al: A risk variant in an miR-125b binding site in BMPR1B is associated with breast cancer pathogenesis. Cancer Res 69: 7459-7465, 2009.

18. Wu Y, Xiao Y, Ding X, et al: A miR-200b/200c/429-binding site polymorphism in the $3^{\prime}$ untranslated region of the AP-2 $\alpha$ gene is associated with cisplatin resistance. PLoS One 6: e29043, 2011.

19. Brendle A, Lei H, Brandt A, et al: Polymorphisms in predicted microRNA-binding sites in integrin genes and breast cancer: ITGB4 as prognostic marker. Carcinogenesis 29: 1394-1399, 2008.

20. Leber B, Geng F, Kale J and Andrews DW: Drugs targeting Bcl-2 family members as an emerging strategy in cancer. Expert Rev Mol Med 12: e28, 2010.

21. Thomadaki H, Talieri M and Scorilas A: Treatment of MCF-7 cells with taxol and etoposide induces distinct alterations in the expression of apoptosis-related genes BCL2, BCL2L12, BAX, CASPASE-9 and FAS. Biol Chem 387: 1081-1086, 2006.

22. Simões-Wüst AP, Schürpf T, Hall J, et al: Bcl-2/bcl-xL bispecific antisense treatment sensitizes breast carcinoma cells to doxorubicin, paclitaxel and cyclophosphamide. Breast Cancer Res Treat 76: 157-166, 2002.

23. Baek D, Villén J, Shin C, et al: The impact of microRNAs on protein output. Nature 455: 64-71, 2008.

24. Michels J, Kepp O, Senovilla L, et al: Functions of BCL-X L at the interface between cell death and metabolism. Int J Cell Biol 2013: 705294, 2013.

25. Billen LP, Kokoski CL, Lovell JF, et al: Bcl-XL inhibits membrane permeabilization by competing with Bax. PLoS Biol 6: e147, 2008.

26. Roush S and Slack FJ: The let-7 family of microRNAs. Trends Cell Biol 18: 505-516, 2008.

27. Boyerinas B, Park SM, Hau A, et al: The role of let-7 in cell differentiation and cancer. Endocr Relat Cancer 17: F19-F36, 2010.

28. O'Day E and Lal A: MicroRNAs and their target gene networks in breast cancer. Breast Cancer Res 12: 201, 2010.

29. Mishra PJ, Mishra PJ, Banerjee D and Bertino JR: MiRSNPs or MiR-polymorphisms, new players in microRNA mediated regulation of the cell: Introducing microRNA pharmacogenomics. Cell Cycle 7: 853-858, 2008.

30. Blitzblau RC and Weidhaas JB: MicroRNA binding-site polymorphisms as potential biomarkers of cancer risk. Mol Diagn Ther 14: 335-342, 2010.

31. Yu Z, Li Z, Jolicoeur N, et al: Aberrant allele frequencies of the SNPs located in microRNA target sites are potentially associated with human cancers. Nucleic Acids Res 35: 4535-4541, 2007.

32. Chen K, Song F, Calin GA, et al: Polymorphisms in microRNA targets: a gold mine for molecular epidemiology. Carcinogenesis 29: 1306-1311, 2008. 